\title{
LOS PROBLEMAS DE LA REFERENCIA: MODELOS PARA LA INTERPRETACIÓN DEL DISCURSO
}

\author{
Francisco José Salguero Lamillar
}

\begin{abstract}
"Cuando veía que ellos [los mayores] decían el nombre de alguna cosa y según aquella voz movían el cuerpo hacia algo, entendía que con aquel vocablo que articulaban también querían mostrar aquel objeto. [...] Así, al oir frecuentemente las palabras empleadas en sus lugares en diferentes frases, comprendía paulatinamente de qué cosas eran signos y, adiestrada a estos signos mi boca, mediante ellos expresaba mis deseos". ${ }^{1}$
\end{abstract}

\section{EL SIGNIFICADO COMO REFERENCIA}

Las más antiguas teorías sobre el significado que se encuentran en la tradición occidental se fundamentan en el poder evocativo de las palabras, habiendo constituido esta facultad que posee el lenguaje natural humano de (re)presentar los objetos ausentes en la mente de los hablantes de una lengua por medio de la voz la base de la teoría del significado durante un buen número de siglos. El texto de Agustín de Hipona que encabeza este artículo es quizás uno de los más representativos de esta concepción que se remonta, al menos, hasta la Stoa antigua, aunque se puede rastrear en otros pensadores anteriores como los megáricos o el propio Platón. En la descripción agustiniana del aprendizaje del significado de los signos, el filósofo africano asume tácitamente la identidad entre significado y referencia, pues la adquisición y desarrollo de la habilidad lingüística depende de la reproducción por parte del hablante de los signos y sus usos, asimilados mediante procedimientos ostensivos frecuentemente repetidos. Esta concepción del significado de los signos lingüísticos se ajusta con agudeza a los rudimentos de una más elaborada teoría de la referencia, que alcanza en la gramática especulativa medieval su expresión más importante antes de la edad contemporánea.

Los gramáticos especulativos desarrollaron al máximo la relación entre signo y referente. Con la teoría de las proprietates terminorum de Pedro Hispano ${ }^{2}$ quedó cerrado el

\footnotetext{
${ }^{1}$ Agustín de Hipona, Confesiones, I, 8.

2 Pedro Hispano, Summulae logicales, libro VII.
} 
triángulo semiótico estoico por su base merced a la distinción entre significatio y suppositio. Esta distinción, que a grandes rasgos y con las debidas precauciones podemos comparar con la distinción más reciente entre connotación y denotación del signo lingüístico, obedecía a la observación de ciertas anomalías en el significado de los términos, entendido este solamente como referencia. Pero con la sustitución de los tratados medievales de lógica en la época moderna y el descrédito casi unánime de la gramática especulativa entre la naciente filología humanista, tal distinción quedó olvidada y hay que remontarse a finales del siglo XIX para que vuelvan a plantearse estas anomalías de la teoría clásica de la referencia desde el punto de vista de una teoría general del significado.

El matemático alemán Gottlob Frege en su célebre artículo "Sobre sentido y referencia"3 es el introductor en la teoría ser lántica actual de esta distinción esencial. Frege, interesado sobre todo por desambiguar el ler guaje de las matemáticas, plantea la siguiente paradoja, llamada de la identidad. En el caso de tener dos términos correferenciales, como por ejemplo "el lucero matutino" y "el lucero vespertino", cualquiera de ellos puede sustituir al otro en un enunciado sin que la verdad (o falsedad) de este varíe. Así, por ejemplo, en el enunciado verdadero

(1) Venus es el lucero matutino

se puede proceder a la sustitución de los términos correferenciales y obtener el enunciado, también verdadero,

(2) Venus es el lucero vespertino.

Asimismo, puede procederse a tal sustitución en la oración enunciativa

(3) El lucero matutino es el lucero vespertino,

obteniéndose el enunciado tautológico

(4) El lucero vespertino es el lucero vespertino.

Entonces, ¿qué diferencia hay entre los enunciados (2) y (4), obtenidos ambos mediante la sustitución de términos correferenciales en los enunciados verdaderos (1) y (3)? Si la sola referencia de los términos implicados es suficiente para determinar su significado y asumiendo que el significado de los enunciados se establece en función del significado de los términos que lo componen ${ }^{4}$, tanto (2) como (4) son enunciados verdaderos y no debe haber ninguna diferencia entre ellos. Pero está claro que mientras que el enunciado (2) aporta información al oyente, del mismo modo que los enunciados (1) y (3), el enunciado (4) no es informativo, por lo que no parece razonable asignarles el mismo significado.

\footnotetext{
${ }^{3}$ G. Frege, "Über Sinn und Bedeutung", Zeitschrift für Philosophie und philosophische Kritik 100 (1892) 25-50. Hay diferentes traducciones al español, como la publicada por Editorial Ariel en G. Frege, Estudios sobre semántica (Barcelona 1984).

${ }^{4}$ A grandes rasgos, esta asunción se corresponde con el denominado principio de composicionalidad de Frege. Ya veremos más adelante de qué tipo de función se trata.
} 
Estas consideraciones llevaron a Frege a proponer la distinción entre la referencia de un término (la suppositio de Pedro Hispano) y su sentido (la significatio). La referencia de los términos "el lucero matutino" y "el lucero vespertino" es la misma (el objeto celeste que conocemos como segundo planeta del sistema solar por su proximidad al Sol y que llamamos Venus), por lo que son términos sinónimos. Sin embargo, su sentido es diferente $\mathrm{y}$, aunque denotan el mismo objeto, pueden distinguirse por la manera como lo denotan, lo que nos hace sospechar que no existen sinónimos perfectos.

El sentido es una condición necesaria para que las expresiones del lenguaje tengan referencia, pero no es condición suficiente, ya que una expresión puede poseer sentido pero carecer de referencia, como por ejemplo el término "el décimo planeta del sistema solar". Por lo tanto, un término tendrá referencia o carecerá de ella en tanto que denote un objeto o no (su referente), es decir: un término tiene referente si denota un objeto real (y entiéndase por real no sólo lo material, sino también abstracciones como "número" o "libertad") y no lo tiene si no denota ningún objeto existente, como en el ejemplo anterior.

La importancia del planteamiento fregeano de esta paradoja radica en que, por primera vez y de forma explícita, se relacionan los problemas del significado de las palabras con el problema del significado de las oraciones en las que se usan y el carácter informativo de estas. Las oraciones enunciativas son para Frege, principalmente, vehículos de información acerca de la realidad, de modo que si en una oración enunciativa aparecen solamente términos referenciales, podrá ser o bien verdadera o bien falsa, dependiendo de lo que se predique del sujeto y de su contraste con la propia realidad, en tanto que si en un enunciado aparece un término sin referente entonces, a pesar de ser gramaticalmente correcto, no será ni verdadero ni falso. En este sentido, Frege opina que la referencia de una oración es su valor veritativo, lo que significa que todas las oraciones verdaderas tienen la misma referencia (lo verdadero) y todas las oraciones falsas también (lo falso), y por lo tanto una oración enunciativa cuyo sujeto, por ejemplo, no tenga un referente tampoco tendrá ella misma referencia, es decir: será una oración asignificativa. ${ }^{5}$ Por tanto, según Frege, el significado oracional depende de lo que habitualmente se conoce como funciones de verdad, cuyo papel en la teoría general del significado pasamos a considerar.

\section{TEORÍA SEMÁNTICA Y FUNCIONES VERITATIVAS}

Es evidente que el significado de los términos constituyentes de una oración no aporta por simple composición el significado de esta. Un ejemplo banal lo aportan las oraciones simples con un verbo transitivo en las que el significado cambia drásticamente si

\footnotetext{
5 Esta idea de que tanto "lo verdadero" como "lo falso" son objetos denotados por los enunciados verdaderos y falsos respectivamente puede parecer bastante extraña en un principio. Pero téngase en cuenta que Frege era un matemático, habituado, por tanto, a tratar con objetos abstractos como parte de la realidad. Por otra parte, el hecho de que todas las oraciones verdaderas ( $\mathrm{y}$ falsas) tengan un mismo referente no debe tampoco hacernos creer que Frege pensaba que no existían diferencias en cuanto a sus significados. Estas diferencias las establece precisamente el sentido de los enunciados.
} 
intercambiamos el sujeto y el objeto. Más interesante son los casos de ambigüedad oracional no producida por ambigüedad léxica, sino por aspectos fundamentalmente sintácticos. Un buen ejemplo lo aporta el siguiente enunciado:

(5) El celoso maltrata a quien ama

La ambigüedad del enunciado (5) depende de las dos interpretaciones, al menos, que un oyente puede darle, sin que medien cambios en el significado de los términos que lo constituyen. Estas dos interpretaciones son las siguientes: ${ }^{6}$

(5') El hombre que es celoso maltrata a todas aquellas personas que él ama,

(5") El hombre que es celoso maltrata a todas aquellas personas que sienten amor.

A pesar de todo, no cabe duda de que el significado oracional es una función del significado de sus términos. La oración se encuentra en un nivel semántico distinto del de las palabras, pero su significado no es independiente del significado de los términos que la componen. Por tanto, una teoría general del significado ha de satisfacer al menos tres condiciones: ${ }^{7}$

$1^{a}$ debe captar en cada lengua la naturaleza del significado de las palabras así como la de las oraciones y explicar la relación que existe entre ambos significados;

$2^{a}$ debe poder predecir la ambigüedad en la interpretación tanto de las palabras como de las oraciones;

$3^{a}$ debe describir y explicar las relaciones sistemáticas que se dan entre las palabras y las oraciones de una lengua, tales como sinonimia, inclusión lógica, vinculación, contradicción, etc.

Las teorías de Frege sobre el significado expuestas de forma somera en la sección anterior constituyen la base de la mayor parte de las teorías semánticas actuales. Junto con la teoría de modelos de Alfred Tarski ${ }^{8}$, la teoría general del significado tiene como pilares fundamentales dos principios que emanan, precisamente, de los trabajos de Frege: el principio de composicionalidad y el principio de extensionalidad.

La teoría de modelos tarskiana ofrece una definición exhaustiva de las condiciones de verdad de las oraciones enunciativas en términos veritativo-funcionales que se ha impuesto en la teoría semántica por su sencillez y por la posibilidad de apelar a modelos algebraicos para interpretar los enunciados del lenguaje. Partiendo de ella, es posible ofrecer una definición del significado oracional que aproveche los modelos algebraicos desarrollados para los lenguajes formales. Se puede considerar que la interpretación semántica de cualquier oración enunciativa gramaticalmente correcta del lenguaje natural remite a la forma lógica de

\footnotetext{
${ }^{6}$ Aún podrían darse otras interpretaciones de (5) según el análisis que se haga de la frase nominal "el celoso", pero dejaremos esa cuestión para otro momento.

7 La siguiente caracterización de la teoría semántica está tomada de R. Kempson, Semantic theory (Londres 1977) 4. Hay traducción española en la Editorial Teide, Teoría semántica (Barcelona 1982).

8 A. Tarski, "Der Wahrheitsbegriff in den formalisierten Sprachen", Studia Philosophica 1 (1936) 261-405. La versión original en polaco es de 1933. Hay traducción al inglés: "The concept of truth in formalized languages" en A. Tarski, Logic, semantics, metamathematics (Oxford 1956) 152-278.
} 
dicha oración. Esta forma lógica es lo que vamos a llamar proposición o también enunciado. Una proposición simple está compuesta por términos individuales, cuya extensión (es decir, su denotación) son objetos del dominio de discurso, y términos predicativos (o más simplemente predicados), cuya extensión son conjuntos ordenados de objetos del dominio de discurso. Las proposiciones complejas se obtienen mediante la aplicación de funciones lógicas cuyos argumentos son proposiciones más simples, denotando siempre alguno de los valores de verdad en función de la extensión de los términos constituyentes y de las funciones lógicas involucradas. A partir de aquí, provistas reglas de correspondencia entre la forma gramatical de una expresión lingüística (la oración) y su forma lógica (la proposición), decir que una oración cualquiera $\boldsymbol{O}$ significa la proposición $\boldsymbol{p}$ (o lo que es lo mismo, establecer el significado de la oración $\boldsymbol{O}$ en relación con su forma lógica) es lo mismo que establecer una relación entre las condiciones que hacen verdadera a la oración $\boldsymbol{O}$ y las condiciones de verdad (definidas como funciones lógico-matemáticas) de la proposición p. De forma abreviada:

(6) $\boldsymbol{O}$ significa que $\boldsymbol{p}={ }_{\text {def. }} \boldsymbol{O}$ es verdadera si y sólo si $\boldsymbol{p} \cdot{ }^{9}$

El principio de composicionalidad es, sin lugar a dudas, el principio más importante de la moderna teoría semántica. Establece que el significado de una expresión compleja es una función de los significados de sus partes y de las reglas sintácticas mediante las cuales se combinan. ${ }^{10}$ De esta definición se desprende la concepción actual según la cual el significado oracional no es independiente, no ya del significado de las palabras que componen la oración, sino tampoco de su sintaxis. Como contrapartida, también el concepto de corrección sintáctica ha cambiado, pasando a depender la corrección de las reglas sintácticas de la posibilidad o no de mostrar una interpretación semántica de las oraciones que se obtienen mediante su aplicación. Sintaxis y semántica se han convertido, por decirlo de un modo gráfico, en las dos caras de una misma moneda. De esta forma, el principio semántico de composicionalidad ofrece una noción más rica y amplia de gramática, en la que la teoría del significado representa un papel importante. ${ }^{11}$

El principio de extensionalidad es más conflictivo. Según este, dadas dos expresiones lingüísticas correferenciales (esto es: con idéntica extensión), ambas son intercambiables en un enunciado salva veritate. ${ }^{12}$ Este principio ofrece problemas, algunos de los cuales quedaron apuntados en el planteamiento de la paradoja de la identidad de Frege. Analicemos estos problemas con cierto detenimiento.

\footnotetext{
${ }^{9}$ Esta definición del significado oracional fue propuesta en primer lugar en D. Davidson, "Truth and meaning", Synthese 17 (1967) 304-323.

${ }^{10}$ B. Partee, A. ter Meulen \& R. Wall, Mathematical methods in linguistics (Dordrecht 1990) 318

11 Cfr. F. J. Salguero Lamillar, "Significado y referencia: hacia una teoría semántica unificada", en E. R. Alcaide, Mª M. Ramos \& F. J. Salguero (eds.), Estudios lingüísticos en torno a la palabra (Sevilla 1993) 217-226.

12 Es decir, sin que cambie su valor de verdad. Este principio se conoce también como regla de sustitución de los idénticos o ley de Leibniz. En realidad, el principio de extensionalidad, tal y como fue expuesto por B. Russell y A. N. Whitehead en Principia Mathematica (Cambridge 1910-1913), se aplica a lenguajes formales, por lo que su definición es algo más compleja.
} 
Es evidente que cuando Frege hace depender el significado oracional de su valor de verdad está pensando principalmente en las oraciones enunciativas declarativas (a las que llamaremos en lo sucesivo enunciados declarativos o, simplemente, enunciados) del tipo de las que conforman una teoría científica, aserciones sobre la realidad como "el quinto planeta del sistema solar es el de mayor tamaño" o "el valor de los ángulos de un triángulo suma 180 grados". Sin embargo, no todas las oraciones significativas del lenguaje son enunciados declarativos. Un ejemplo evidente es el de las oraciones imperativas, de las que difícilmente se puede decir que sean verdaderas o falsas, sin que por ello caigan en la categoría de oraciones asignificativas. Pero aun entre los enunciados declarativos cabe encontrar problemas de interpretación según esta concepción. Sea por ejemplo el enunciado

(7) Copérnico creía que las órbitas de los planetas son circulares.

En (7), la oración subordinada

(8) Las órbitas de los planetas son circulares

es un enunciado declarativo falso. Teniendo en cuenta que el enunciado (7) es verdadero en su conjunto, también debería serlo cualquier enunciado que se obtenga al sustituir la cláusula subordinada falsa por otra de idéntico valor de verdad, como puede ser el caso del enunciado falso

(9) Dos y dos suman cinco

obteniéndose el enunciado

(7') Copérnico creía que dos y dos suman cinco.

Pero sin duda el enunciado ( $7^{\prime}$ ) es falso, contrariamente a lo que habíamos supuesto, por lo que nos encontramos con un verdadero problema concerniente a la correferencialidad de los enunciados (8) y (9), al menos por lo que respecta a su intersustituibilidad en (7).

El problema se ve más claro, quizás, cuando son términos y no oraciones enunciativas lo que se sustituye. Considérese, por ejemplo, el enunciado

(10) Juan cree que Ulises fue rey de Ítaca.

A pesar de que, efectivamente, entre las creencias de Juan se encuentre la de que Ulises, el héroe homérico, reinó en la isla de Ítaca, siendo por tanto (10) un enunciado verdadero, no es necesario que el enunciado

(11) Juan cree que Odiseo fue rey de Ítaca,

obtenido a partir de (10) mediante la sustitución de los términos correferenciales "Ulises" y "Odiseo", sea verdadero también, ya que puede ocurrir que Juan no sepa a quién se refiere el nombre "Odiseo".

El principio de extensionalidad falla aquí también, por lo tanto, pues predice que si (10) es verdadero también debe serlo (11). Este fallo no se da en todos los casos (hay multitud de ejemplos en los que el principio de extensionalidad funciona), sino en determinados 
contextos denominados referencialmente opacos ${ }^{13}$. En nuestros ejemplos, el verbo "creer" introduce uno de estos contextos opacos. En general, en los lenguajes naturales, estos contextos están introducidos por ciertos adverbios y giros lingüísticos, como "necesariamente", "obligatoriamente", "es posible", "está prohibido", etc.; por los denominados verbos de actitud proposicional, verbos completivos del mismo tipo de "creer" como por ejemplo "saber", "dudar", "suponer", etc.; por ciertos verbos transitivos, como "querer", "buscar", "necesitar", "percibir", etc. Los lenguajes en los que existen contextos referencialmente opacos se conocen como lenguajes intensionales.

\section{MUNDOS POSIBLES, ESTADOS DE INFORMACIÓN Y BASES DE DATOS}

En los lenguajes intensionales, la referencia de los términos no es suficiente para establecer el valor de verdad de los enunciados que los contienen, por lo que se hace imprescindible determinar también su sentido. En el caso del enunciado (11), el que este sea verdadero o falso depende, evidentemente, de las creencias de Juan. Hemos supuesto que Juan no cree que Odiseo reinase alguna vez en Ítaca a pesar de que cree que Ulises sí lo hizo, pero del mismo modo podemos pensar en un estado de cosas diferente por lo que respecta a las creencias de Juan en el que este identifique a Odiseo con Ulises, en cuyo caso (11) será un enunciado verdadero. Lo mismo ocurre con el enunciado (7). Podemos imaginar un mundo en el que Copérnico se hubiese adelantado a Kepler en el establecimiento de las leyes de traslación de los planetas alrededor del Sol, con lo que la historia de la astronomía habría cambiado sustancialmente. Estos mundos posibles no son sino interpretaciones alternativas de los enunciados de los lenguajes intensionales que nos permiten definir en términos de teoría de modelos el sentido de las expresiones del lenguaje del mismo modo que antes definimos su referencia apelando a la extensión de los términos. De aquí que digamos que el sentido de una expresión lingüística no es nada más que todas las extensiones (denotaciones) variables que la expresión puede tener, organizadas como una función con todos los posibles estados de cosas como argumentos y las extensiones apropiadas dispuestas como valores. ${ }^{14}$

La noción de estados de cosas o mundo posible fue acuñada por Rudolf Carnap ${ }^{15}$, quien la introdujo en el ámbito de la lógica mediado el presente siglo. Pero su importancia en la teoría semántica actual se debe, sobre todo, al papel que representa en la definición del sentido en teoría de modelos y al uso que de ella hizo Richard Montague en su análisis formal de ciertos fragmentos del inglés. ${ }^{16}$ En el lenguaje intensional de Montague se mantiene el principio de composicionalidad de Frege, de modo que su gramática del inglés parte de dos ideas fundamentales:

\footnotetext{
13 W. O. Quine, Word and objêct (Cambridge, Mass. 1960).

14 D. R. Dowty, R. E. Wall \& S. Peters, Introduction to Montague semantics (Dordrecht 1981) 145.

15 R. Carnap, Meaning and Necessity (Chicago 1947).

16 R. Montague, Formal Philosophy. Selected papers of Richard Montague (Londres 1974).
} 
$1^{\text {a }}$ El significado del todo es función del significado de las partes.

$2^{\mathrm{a}} \mathrm{La}$ sintaxis del lenguaje está esencialmente conectada con la semántica, de forma que la sintaxis muestra cómo han de combinarse los significados.

En la teoría del significado montagoviana, por tanto, la forma gramatical de una expresión y su forma lógica están indisolublemente unidas, cumpliendo el requisito formulado anteriormente de aportar reglas de correspondencia entre la oración y su forma lógica para un fragmento del inglés. ${ }^{17}$ De este modo, la teoría semántica de Montague es también una teoría gramatical. Su gramática es una gramática formalizada en la que a partir de un conjunto de principios y reglas de transformación se obtienen las oraciones gramaticalmente correctas y significativas del lenguaje como si fuesen sus teoremas.

A partir de dos categorías básicas, la categoría que representa los soportes lingüísticos de la referencia (los términos individuales), representada por el tipo categorial $e$, y la que representa los soportes de la verdad (los enunciados), representada por el tipo categorial $t$, Montague define el resto de las categorías sintácticas. A estas dos categorías básicas ha de añadirse la categoría que representa los sentidos de los diferentes tipos gramaticales a que dan lugar las dos categorías anteriores, es decir: un conjunto de índices o mundos posibles representativos de los diferentes estados de cosas en los que las expresiones del lenguaje pueden tener su extensión, representada por el tipo categorial $s$. A través de ellas puede asignarse un tipo categorial a cualquier parte de la oración y establecerse mediante la teoría de modelos el significado de esta.

Los mundos posibles pueden reinterpretarse en el seno de una teoría general de la información como estados de conocimiento del hablante/oyente en el proceso de comunicación o bien como estados temporales de la máquina. En general, esto es lo que se conoce como estados de información. La idea central de la teoría general del significado aplicada a la teoría de la información es que el valor de verdad de los enunciados emitidos por un hablante depende de dichos estados de información. Si se define un orden parcial entre estados de información, similar a la relación de accesibilidad entre mundos posibles, un estado de información cualquiera contendrá tanto la información de los enunciados que lo componen cuanto la derivada lógicamente de enunciados pertenecientes a estados de información "temporalmente" anteriores, así como la información que se desprende de sus enunciados, la cual puede pasar a formar parte de estados de información "temporalmente" posteriores. ${ }^{18}$

Estos estados de información pueden ser, por ejemplo, bases de datos léxicas aportadas en el proceso de comunicación por un hablante a un oyente. Cada una de estas bases de datos contiene toda la información léxica disponible en un cierto momento para el

\footnotetext{
17 Cfr. R. Montague, "English as a formal language" en (Montague 1974:188-221) y "The proper treatment of quantification in ordinary English" en (Montague 1974:247-270).

18 Este planteamiento es, básicamente, lo que se conoce como semántica de Kripke, en honor a Saul Kripke, quien a partir de esta idea fundamental desarrolló una interpretación intuicionista de la lógica de predicados. Cfr. S. Kripke, "Semantic analysis for intuitionistic logic I", en M. Dummet \& J. Crossley (eds.), Formal systems and recursive functions (Amsterdam 1965) 92-129.
} 
hablante/oyente. Esta información consta, principalmente, de una etiqueta léxica, su correspondiente tipo categorial de la clase de los usados por Montague para su gramática del inglés y, a veces, una relación de orden de los elementos de la base de datos. De modo que, dada una base de datos de este tipo, es posible manipular la información que contiene mediante reglas lógicas e incrementar esta información. Cuando el hablante codifica el mensaje, podemos imaginar que su labor consiste, de hecho, en escoger las entradas léxicas relevantes a partir de una base de datos léxica común y combinarlas según sus propiedades combinatorias declaradas por su tipo categorial. El oyente interpreta los datos que obtiene a partir del hablante como miembros de una base de datos homogénea y aplica todas sus capacidades deductivas con el fin de obtener la mayor cantidad posible de la información codificada en el mensaje original. Pero a veces, el oyente necesita apelar a más de un grupo de datos homogéneos para obtener la información, como en el caso de los enunciados en los que aparecen contextos opacos o aquellos en los que aparecen expresiones cuya referencia no depende de las expresiones mismas, sino de otras expresiones del discurso. Este último tipo de expresiones es lo que conocemos como expresiones anafóricas o anáforas. Esto significa que en el proceso de decodificación del mensaje, al determinar el significado de una expresión no puramente referencial, como es el caso del término "Odiseo" en el enunciado (11), o el del pronombre "él" en el enunciado

(12) Cada atleta piensa que él será el vencedor,

el oyente ha de considerar otras relaciones además de las estrictamente semánticas, tales como ciertos aspectos sintácticos y de cohesión del discurso, así como también aquellas presuposiciones que tanto hablante como oyente asumen acerca de la referencia de los términos que aparecen en el discurso y que se hallan más cerca de los aspectos pragmáticos de la comunicación que de los propiamente semánticos.

\section{FRASES NOMINALES, CUANTIFICACIÓN Y ANÁFORA}

En el enunciado

(13) Un hombre entró en la habitación

la frase nominal "un hombre" es una expresión anafórica. Sin embargo, un análisis adecuado de su forma y de las relaciones gramaticales que mantiene con el resto de los términos del enunciado le dice al oyente cómo ha de buscar su referencia en las bases de datos disponibles. La forma lógica de (13) esclarece su interpretación:

(13') $\exists x(\operatorname{HOMBRE}(x) \wedge$ ENTRAR_EN(x,la_habitación))

En (13'), $\exists$ es un cuantificador existencial que liga a la variable individual $x$, el signo $\wedge$ es la conjunción lógica, los términos en versalitas HOMBRE y ENTRAR_EN son dos predicados, monádico el primero (admite un solo argumento) y diádico el segundo (admite dos argumentos a los que sirve de relación). Por simplicidad se han desestimado tiempo y 
aspecto del enunciado y el análisis de la frase nominal "la habitación", que sería similar al análisis de "un hombre". 19

En primer lugar, hay una presuposición de existencia en la expresión, lo que impide que interpretemos la frase nominal como una expresión vacía de contenido. Lo que no hay en $\left(13^{\prime}\right)$, sin embargo, es una función de individualidad, por lo que el oyente ha de interpretar la expresión de una forma genérica, en principio. Esta generalidad la componen todas las expresiones de tipo $e$ que formen parte de la base de datos léxica original a la que remite el hablante y a las que convenga el predicado HOMBRE; esto es: todos aquellos nombres que al sustituir a la variable $x$ en el enunciado $\operatorname{HOMBRE}(\mathrm{x})$ hagan de este un enunciado verdadero, según un modelo algebraico definido previamente.

En general, las frases nominales construidas con un determinante y un nombre común son descripciones del objeto o conjunto de objetos del discurso que la frase denota. El establecimiento de su referencia depende de procesos lógicos relacionados con la cuantificación, por lo que podemos decir que la interpretación correcta de los determinantes de las lenguas naturales es como cuantificadores que introducen variables dependientes, también denominadas variables ligadas.

Otro tanto ocurre con la interpretación del enunciado (12) de la sección anterior:

(12) Cada atleta presiente que él será el vencedor.

La frase nominal "cada atleta" introduce una variable ligada, en esta ocasión por un cuantificador universal. Pero (12) plantea otros problemas de referencialidad aparte de la frase nominal. El verbo "presentir" es un verbo de actitud proposicional como "creer", por lo que introduce un contexto opaco. Además de eso, el pronombre "él" es una expresión anafórica que bien puede estar ligada al cuantificador universal introducido por la frase nominal sujeto, bien puede estar libre en su contexto y, por lo tanto, remitir a un referente no relacionado con dicha frase nominal. Esto explica las dos interpretaciones posibles de (12), que se ven más claramente si se analizan sus respectivas formas lógicas:

$$
\begin{aligned}
& \left(12^{\prime}\right) \forall x\left(\operatorname{ATLETA}(x) \rightarrow\left(\operatorname{PRESENTIR}{ }_{\mathrm{X}}\right)\left(\text { SER_EL_VENCEDOR }_{\mathrm{N}} \mathrm{x}\right)\right)^{20} \\
& (12 ") \forall \mathrm{x}\left(\operatorname{ATLETA}(\mathrm{x}) \rightarrow \exists \mathrm{y}\left(\operatorname{ATLETA}(\mathrm{y}) \wedge\left(\operatorname{PRESENTIR}_{\mathrm{X}}\right)\left(\text { SER_EL_VENCEDOR}_{\mathrm{N}} \mathrm{y}\right)\right)\right)^{21}
\end{aligned}
$$

Las formas lógicas de (12) y (13) nos permiten desentrañar los problemas de referencialidad implicados en ambos enunciados, remitiendo su solución a modelos algebraicos. Sin embargo, tanto (12') como (12") como (13') esconden las relaciones

19 El enunciado (13') puede leerse del siguiente modo: "Hay al menos (existe) un individuo tal que a ese individuo le conviene el predicado HOMBRE y ese individuo se halla en la relación ENTRAR_EN con el individuo denotado por el término 'la_habitación'".

${ }^{20}$ El signo $\forall$ es el cuantificador universal que liga a la variable $x$, mientras que $\rightarrow$ es el signo correspondiente a la implicación lógica, pudiéndose leer (12') como "Para todo objeto $x$, si a $x$ le conviene el predicado ATLETA entonces $x$ presiente que a $x$ le conviene el predicado SER_EL_VENCEDOR". (PRESENTIR ${ }_{\mathrm{X}}$ ) es un operador de modalidad.

${ }^{21}$ Es decir: "Para todo objeto $x$, si a $x$ le conviene el predicado ATLETA entonces existe un objeto $y$ tal que a $y$ le conviene el predicado ATLETA y $x$ presiente que a $y$ le conviene el predicado SER_EL_VENCEDOR". 
gramaticales no semánticas que se dan entre los términos de (12) y (13) al reemplazarlas por relaciones lógicas. Esto puede ser indeseable para determinados tipos de análisis. La solución consiste en reemplazar la cuantificación lógica habitual por una abstracción sobre la variable, abstracción que define un conjunto de individuos. Esto es lo que se conoce habitualmente como $\lambda$-abstracción. De este modo, (13') puede reescribirse:

(13") $\lambda x[\operatorname{HOMBRE}(\mathrm{x}) \wedge \text { ENTRAR_EN(x,la_habitación) }]^{22}$.

La diferencia entre las interpretaciones (13') y (13") es que la primera es una proposición que declara la forma lógica de (13), mientras que la segunda es un predicado monádico, cuyo tipo categorial es $\langle e, t\rangle$, que puede predicarse de cualquier expresión de tipo $e$ que aparezca en las bases de datos disponibles para el hablante/oyente, de modo que mediante una simple regla de aplicación de ambos tipos categoriales obtendríamos una expresión de tipo $t$, es decir, un enunciado en el que la variable $x$ sería sustituida por la expresión de tipo $e$ de la que se predique (13"). Esta expresión, evidentemente, constituiría la referencia de la frase nominal "un hombre".

Una explicación similar puede darse para la ambigüedad del enunciado (5) que aparece como ejemplo en la sección 2:

(5) El celoso maltrata a quien ama.

Sus dos interpretaciones (5') y (5") son las expresiones correspondientes a las siguientes proposiciones:

$$
\begin{aligned}
& \text { (5') } \forall \mathrm{x}(\operatorname{CELOSO}(\mathrm{x}) \rightarrow \forall \mathrm{y}(\operatorname{Ama}(\mathrm{x}, \mathrm{y}) \rightarrow \operatorname{Maltrata}(\mathrm{x}, \mathrm{y}))) \\
& \left(5^{\prime \prime}\right) \forall \mathrm{x}(\operatorname{CELoso}(\mathrm{x}) \rightarrow \forall \mathrm{y} \exists \mathrm{z}(\operatorname{Ama}(\mathrm{y}, \mathrm{z}) \rightarrow \operatorname{MaLtrata}(\mathrm{x}, \mathrm{y})))
\end{aligned}
$$

En realidad, las formas lógicas de ambas interpretaciones del enunciado (5) se corresponden con los dos análisis siguientes en el marco G.B.:

(5a) $\left[_{\mathrm{SNS}}\right.$ El celoso] [SVP maltrata [SNOD a quien [ $h_{\text {el_celoso }}$ ama $\left.\left.\left.h_{\text {quien }}\right]\right]\right]$

(5b) [SNS El celoso] [SVP maltrata [SNOD a quien [ $h_{\text {quien }}$ ama $\left.\left.\left.h\right]\right]\right]$

En (5a), el pronombre "quien" se comporta como un operador cuantificacional que liga la huella $h_{\text {quien. }}$ Esta huella es una variable que se comporta sintácticamente como objeto directo del verbo amar, correspondiéndose con el segundo argumento del enunciado $\operatorname{AMA}(\mathrm{x}, \mathrm{y})$ en $\left(5^{\prime}\right)$, en tanto que la huella $h_{\mathrm{el}}$ celoso es una anáfora ligada a la frase nominal "el celoso" y hace la función de sujeto del verbo amar, correspondiéndose con el primer argumento del enunciado $\operatorname{AMA}(\mathrm{x}, \mathrm{y})$ en $\left(5^{\prime}\right)$.

En el análisis (5b), en cambio, la huella $h_{\text {quien }}$ hace la función de sujeto del verbo amar, mientras que su objeto directo, la huella $h$, es una anáfora que no encuentra su referencia dentro del enunciado (5). Esto se corresponde con el orden de las variables ligadas que son los argumentos del enunciado $\operatorname{AMA}(\mathrm{y}, \mathrm{z})$ en (5").

22 Es decir: "Los $x$ tales que a $x$ le conviene el predicado HOMBRE y $x$ se halla en la relación ENTRAR_EN con el objeto denotado por el término 'la_habitación'". 
De esta forma, a partir del análisis de la ambigüedad de (5), queda patente la estrecha relación que existe entre sintaxis y semántica cuando se trata de dar explicación de los problemas que surgen en la interpretación de los enunciados por un oyente cualquiera. Esta relación queda perfectamente asumida por la gramática categorial, que ofrece la posibilidad de crear bases de datos léxicas en las que cada entrada es un miembro de un sistema lógico estructurado en el que toda la información semántica y sintáctica (tipos categoriales) aportada por el léxico es utilizada por el hablante (oyente) para codificar (decodificar) la información pertinente en cada proceso comunicativo. ${ }^{23}$

Los problemas de referencialidad del lenguaje natural quedan resueltos, por tanto, en un marco amplio de procesamiento de la información de los procesos comunicativos lingüísticos. Esto abre nuevas perspectivas en el estudio de la semántica, convirtiéndose sintaxis, semántica y pragmática en un continuum semiótico en el que sus fronteras quedan, cuanto menos, difuminadas.

\footnotetext{
23 Un sistema lógico estructurado de este tipo ha sido desarrollado por Dov Gabbay, Labelled Deductive Systems (Oxford, en prensa). Los sistemas deductivos etiquetados son un marco computacional general de manejo de bases de datos. Sobre esta base es posible hacer un análisis categorial del lenguaje natural como el presentado aquí. En este sentido, cfr.: D. Gabbay \& R. Kempson, "Natural-Language content and information flow: a proof theoretic perspective" (Londres 1992); F. J. Salguero Lamillar, "Una aproximación categorial al análisis de la interfaz sintaxis-semántica en el marco de los Sistemas Deductivos Etiquetados", en C. Martín Vide (ed.), Actas del VIII Congreso de Lenguajes Naturales y Lenguajes Formales (Barcelona 1992); F. J. Salguero Lamillar, "Anaphoric instantiation problems in an inferential model of utterance representation", en C. Martín Vide (ed.), Current Issues in Mathematical Linguistics (Amsterdam 1994).
} 